\title{
INTERRUPCIONES Y SOLAPAMIENTOS EN EL DISCURSO ORAL DE HABLANTES CON Y SIN SÍNDROME DE ASPERGER*
}

\author{
INTERRUPTIONS AND OVERLAPS IN THE ORAL SPEECH OF \\ SPEAKERS WITH AND WITHOUT ASPERGER SYNDROME
}

FRANCISCO J. RODRÍGUEZ MUÑOZ

Universidad de Almería

frodriguez@ual.es

\section{RESUMEN}

Este estudio atiende a las diferencias cuantitativas y cualitativas a propósito de las interrupciones y los solapamientos que se producen en el discurso oral de 20 sujetos con síndrome de Asperger y 20 con desarrollo típico. Asimismo, ofrece una clasificación para el análisis discursivo de la interrupción acogiéndose a los principios de cooperación y gestión temática. De acuerdo con los resultados, desde un punto de vista cuantitativo, ambos tipos de transiciones entre turnos son significativamente más numerosos en la muestra patológica, y desde una perspectiva cualitativa, las interrupciones son menos relevantes y cooperativas en el mismo grupo. En suma, se aportan signos sobre el comportamiento comunicativo de los hablantes con síndrome de Asperger y, en consecuencia, se sugiere el refuerzo de la relevancia y la cooperación en sus intercambios comunicativos.

Palabras clave: Discurso oral, interrupción, síndrome de Asperger, solapamiento.

\section{ABSTRACT}

This study pays attention to the quantitative and qualitative differences in relation to

"Esta investigación es parte de la tesis doctoral titulada Los déficits pragmáticos en el discurso oral de niños con sindrome de Asperger, dirigida por los doctores Bañón Hernández y Fornieles Alcaraz (Universidad de Almería), con Mención Internacional y subvencionada por el Programa FPU (AP2008-01707) del Ministerio de Educación, Cultura y Deporte (Gobierno de España). Además, el estudio se inscribe en el proyecto Ampliación y adaptación del corpus PERLA de datos clinicos en el marco del proyecto internacional Childes/Talkbank: perfiles pragmáticos y propuestas de intervención (FFI2012-39325-C03-01). Agradecemos la inestimable colaboración de las asociaciones asturiana y valenciana de síndrome de Asperger, del Departamento de Orientación Psicopedagógica del IES Argar (Almería) y de todos los participantes implicados en el trabajo. 
interruptions and overlaps that are produced in the oral speech of 20 subjects with Asperger syndrome and 20 with typical development. Likewise, it offers a classification for the discursive analysis of interruption making use of the cooperation and topic management principles. According to results, from a quantitative point of view, both types of turn transitions are significantly more numerous in the pathological sample, and from a qualitative perspective, interruptions are less relevant and cooperative in the same group. In short, signs about the communicative behaviour of speakers with Asperger syndrome are provided and, in consequence, the reinforcement of relevance and cooperation in their communicative exchanges is suggested.

Keywords: Oral speech, interruption, Asperger syndrome, overlap.

Recibido: 22.08.2014. Aceptado: 27.01.2015.

\section{INTRODUCCIÓN}

- 1 presente estudio tiene por objeto analizar dos tipos de transiciones entre turCnos conversacionales -las interrupciones y los solapamientos- en el discurso oral de hablantes con y sin síndrome de Asperger (SA). Las características de este trastorno se definen, en líneas generales, por las dificultades en las relaciones interpersonales y en la comunicación verbal y no verbal. De hecho, el autor piensa que muchas de las causas que determinan el fracaso o la limitación en las relaciones sociales que protagonizan tales sujetos hay que buscarlas en su comportamiento comunicativo y son de naturaleza eminentemente interactiva ( $c f$. Rodríguez $\mathrm{Mu}$ ñoz, 2012, 2013).

Los supuestos de los que parte esta investigación se resumen en: i) un predominio cuantitativamente significativo de solapamientos e interrupciones de distinto tipo por parte de los hablantes con SA, frente al grupo control; y ii) en el marco taxonómico que se establece para la interrupción, se prevé que serán menos cooperativas y menos relevantes en el caso de los sujetos diagnosticados con SA.

Tradicionalmente, las interrupciones han sido interpretadas como indicadores de objeción y se han asociado a las estrategias de dominación discursiva. Como función elemental de la interrupción, a menudo se ha sostenido que a través de ella es posible obtener el control inmediato del discurso, del turno o de los temas conversacionales. Así pues, las interrupciones ejercen presión sobre el interlocutor para que éste renuncie al control de la interacción y ceda así el espacio correspondiente a su turno conversacional.

Adoptando el enfoque precedente, según el cual la interrupción es concebida como una estrategia interactiva de poder e intromisión en el terreno discursivo ajeno, autores como Farina (1960), Hetherington et al. (1971), Jacob (1974, 1975), Kollock et al. (1985), Meltzer et al. (1971), Mishler y Waxler (1968), Orcutt y 
Harvey (1985), Riskin y Faunce (1972) y West (1979), por citar algunos, sitúan sus investigaciones de corte lingüístico y, en ocasiones, más psicológico y sociológico.

La postura anterior ha sido cuestionada por un gran número de estudios que determinan que las correlaciones entre la interrupción y las estrategias de dominación, control y poder discursivos son más débiles de lo que en un principio pudiera parecer. Esta es una de las premisas de las que parten trabajos como los de Beattie (1981, 1982), Bennett (1981), Dindia (1987), Edelsky (1981), ErvinTripp (1979), Ferguson (1977), French y Local (1983), Gallois y Markel (1975), Houtkoop y Mazeland (1985), Kennedy y Camden (1983), Murray (1987) y Tannen (1981). Para dichos autores, algunas interrupciones cumplen otro tipo de funciones comunicativas, como la petición de aclaración de información o la necesidad de completarla por parte del que interrumpe. En tales supuestos, el interlocutor no pretende ganar terreno, sino que muestra interés por la intervención de su colocutor y una implicación activa en el discurso de éste.

Algunas investigaciones más recientes que se han ocupado del análisis de la interrupción han sido las de Bogetic (2011), en narraciones colaborativas; Burgos (2007), para quien las interrupciones pueden constituir, en efecto, actos de cooperación conversacional; Farley et al. (2010), de acuerdo con las reacciones no verbales que generan; Menz y Al-Roubaie (2008), en interacciones médico-paciente; O’Reilly (2008), en procesos terapéuticos familiares; entre otros.

En este estudio rescatamos el modelo propuesto por Goldberg (1990), quien clasifica los tipos de interrupción de acuerdo con su función estratégica y su rentabilidad comunicativa en: interrupciones orientadas al poder, interrupciones orientadas al objeto de la comunicación e interrupciones neutras. Retomando el principio griceano de cooperación conversacional (Grice, 1975) y la distinción que lleva a cabo Gallardo (1993) entre solapamientos competitivos y colaborativos, en esta investigación renombraremos los tipos de interrupciones según sean cooperativas, no-cooperativas o neutras, sumándonos a los planteamientos que subyacen en otros análisis como el de Murata (1994).

Las interrupciones encaminadas al poder - para el autor, no-cooperativas- implican una intervención intrusiva, inapropiada o descortés y comportan inserciones que no son coherentes con los comentarios del interlocutor que es interrumpido. Por lo tanto, transmiten aversión, antipatía y, en el peor de los casos, constituyen un ataque hostil hacia el hablante y representan un acto conflictivo. Además, implican a menudo un cambio temático o la recuperación de algún tema que fue tratado con anterioridad en el discurso. Con todo, se define este grupo a partir del concepto de intrusión discursiva; esto es, el conjunto de mensajes interruptores que tienen en común ser poco coherentes con el tema en curso e imponer un obstáculo al desarrollo fluido del intercambio comunicativo.

Las interrupciones que se orientan al objeto de la interacción -las coopera- 
tivas- están basadas en la compenetración comunicativa y constituyen actos de habla colaborativos. Este tipo de interrupción implica interés con respecto a la intervención del interlocutor interrumpido y constituye una estrategia útil para expresar afecto, empatía o solidaridad en relación con el mensaje que se expresa en el turno anterior. Las interrupciones cooperativas contribuyen, pues, al desarrollo de la interacción oral, cubriendo huecos de información, (re)elaborando el tema que es objeto de la interrupción, introduciendo comentarios, juicios valorativos o solicitando al interlocutor un mayor número de datos sobre el tema del que trata en su turno conversacional.

Las interrupciones que contemplamos en tercer lugar -las neutras- se dirigen siempre a las necesidades inmediatas que exige una situación comunicativa determinada. Suelen dar lugar a una repetición o a la reparación conversacional y, con frecuencia, reclaman al interlocutor una aclaración de la emisión precedente. Del mismo modo, pueden referirse a un acontecimiento o a un asunto externo a la enunciación que requiere atención por parte de los interlocutores antes de que la interacción prosiga. Una vez resuelto dicho "accidente conversacional", el discurso retorna al estado previo a la interrupción, permitiendo al interlocutor que fue interrumpido continuar por el lugar donde se quedó. No están orientadas al dominio discursivo ni tampoco están directamente relacionadas con el objeto de la conversación. De acuerdo al autor, esta clase de interrupciones suele relacionarse con los "ruidos" que pueden aparecer en el transcurso de la interacción y que, en ciertas ocasiones, se resuelven por medio de la interrupción.

\subsection{Gestión temática en los procesos de interrupción}

Esta propuesta teórico-metodológica pretende integrar, en el marco de la tipología básica que se concibe para la interrupción en este análisis (cooperativas, no-cooperativas y neutras), las relaciones semánticas que se establecen entre las interrupciones que realizan los hablantes. De esta forma, se podrá juzgar si los hablantes con y sin SA que participan en el estudio son capaces de establecer una comunicación eficaz sobre el tema del que hablan y contribuir a éste con sus interrupciones.

Un modelo que resume de forma completa y sistemática los conceptos de referencia en la dinámica temático-informativa es el que plantea Bañón (1997). Los principios que aquí se tienen en cuenta son:

a. Actitud: de acuerdo con el tema, la interrupción puede ser transformadora o conservadora. Dependiendo de los argumentos, se considera coincidente o discrepante.

b. Previsibilidad: según la probabilidad de aparición del mensaje interruptor, se 
puede distinguir las interrupciones previsibles de las imprevisibles, y de acuerdo con el momento en que se produce la interrupción, las temáticas de las remáticas.

c Relevancia: teniendo en cuenta la conveniencia del mensaje interruptor, las interrupciones pueden ser pertinentes o impertinentes, y según la repercusión que el mensaje interruptor tenga sobre el discurso interrumpido, trascendentes o intrascendentes.

d. Oportunidad: las interrupciones pueden ser contiguas o inmediatas o, por el contrario, no-contiguas o mediatas.

Simplificando el modelo anterior y adaptando el esquema propuesto por Dunn y Shatz (1989), quienes distinguen hasta seis categorías semánticas relacionadas con la interrupción, se atenderá a tres de las condiciones que pueden presentar:

a. Relevancia: se diferencian tres tipos de aportaciones más o menos pertinentes que el mensaje interruptor puede llevar aparejadas. Se asume que una interrupción es relevante cuando el mensaje interruptor está asociado semánticamente al turno inmediatamente anterior -en este caso, siempre es de un adulto (E)- y viene al caso. Una interrupción será semirrelevante cuando el mensaje interruptor sea pertinente con los turnos previos del niño (con o sin SA), pero sin guardar relación con el inmediato anterior del adulto. Por último, son irrelevantes aquellos mensajes interruptores que no mantienen vinculación con ninguna idea expuesta previamente.

b. Estatus informativo: la información que contiene el mensaje interruptor puede ser nueva (información que no fue mencionada en turnos anteriores) o antigua (esencialmente, repeticiones o rescate de información previa). Un estado informativo nuevo supone un avance temático, mientras que un estado antiguo implica retomar, sin ningún matiz, información proporcionada con anterioridad en la conversación. Se denominará remáticas a aquellas interrupciones que introducen datos nuevos, y temáticas, a las que recuperan información proporcionada con anterioridad.

c. Éxito: cuando el adulto responde al niño directamente o hace referencia a la intervención del niño en el siguiente turno de habla, se considera que la interrupción ha sido exitosa; en cambio, si la intervención es ignorada y, simplemente, no es atendida por el adulto, se juzga que es no-exitosa. Por norma, en los supuestos a los que Gallardo (1993) denomina, a partir de Ferguson (1977), "amago de interrupción" -esto es, cuando tiene lugar el habla simultánea sin que uno de los interlocutores llegue a conseguir la palabra y la ruptura de continuidad sucede en el mismo turno que intentaba interrumpir-, se entiende que la interrupción nunca tiene éxito. En otras palabras, coincidiendo con Gallardo (1993), se trata de una "interrupción que no triunfa". 
En ocasiones, las interrupciones vienen expresadas por estructuras ilativas y continuadoras del discurso que permiten el progreso de los turnos ( $c f$. Bañón, 1997); por ejemplo, pues o entonces. En tales casos, evaluamos la relevancia en función del mensaje no interruptor que sucede a la interrupción breve. Asimismo, en estas circunstancias, el estatus informativo es valorado en relación con los datos que proporciona el interlocutor en el mensaje que acontece tras la interrupción. El éxito variará, igualmente, según la aceptación que tenga el mensaje no interruptor pospuesto a la interrupción.

\subsection{Solapamientos}

Con cierta frecuencia, el entrevistador o el entrevistado pronuncian un sonido fático o interjecciones del tipo uhum, ajá u otras marcas conversacionales como vale, para garantizar que la información ha sido recibida. En tales casos, los hablantes tienden a iniciar su intervención con simultaneidad a la emisión de dichas palabras.

No se puede considerar que se esté, pues, ante interrupciones stricto sensu; razón por la cual se ha decidido tratarlas como solapamientos, con independencia de que haya continuidad o no en las emisiones, ya que no expresan ningún contenido semántico-proposicional preciso. Dicho de otro modo, el solapamiento no constituye, para el autor, una estrategia orientada a ganar terreno conversacional. Gallardo $(2003,2006)$ se percata de que, en efecto, existen unidades limítrofes que ocupan una "posición bisagra"; se trata de actos de habla dinámicos o de enlace cuyo solapamiento no suele plantear ningún problema. Liddicoat (2007) adopta una postura similar al sostener que, a menudo, aparecen solapamientos breves en la interacción oral que no son tratados como interrupciones por los participantes, puesto que casi nunca son problemáticos.

En resumen, para el autor la distinción más básica se establece entre solapamientos e interrupciones. Por lo general, para el autor la concepción de ambas categorías es deudora de los principios que expone Ferguson (1977). Por consiguiente, el solapamiento no constituye -tampoco para el autor- la ruptura de la continuidad de la emisión del hablante anterior; en cambio, la interrupción simple sí supondría romper con dicha continuidad. Este es uno de los principales criterios que se asume en este análisis al diferenciar ambas clases de transiciones entre turnos.

Seguidamente, en la clasificación de la interrupción se marca dos niveles de análisis, como resume la Tabla I. Por un lado, una distinción principal es la que se establece a partir del principio de cooperación, que está relacionada con la construcción de la imagen social de los hablantes. Esto permite hablar de interrupciones cooperativas, no-cooperativas y neutras. Por otro lado, se tiene en cuenta tres 
categorías asociadas al carácter del mensaje interruptor en el proceso de gestión temática: la relevancia (mensaje interruptor relevante, semirrelevante o irrelevante), el estatus informativo (mensaje interruptor con información nueva o antigua) y el éxito (mensajes interruptores exitosos o no-exitosos).

Tabla I. Taxonomía y caracterización de la interrupción para su análisis conversacional.

\begin{tabular}{|c|c|c|}
\hline \multicolumn{3}{|c|}{ COOPERACIÓN CONVERSACIONAL } \\
\hline Cooperativas & No-cooperativas & Neutras \\
\hline $\begin{array}{l}\text { Actos de habla } \\
\text { colaborativos. } \\
\text { Se basan en el objeto } \\
\text { de la conversación y } \\
\text { en la compenetración } \\
\text { comunicativa. } \\
\text { Interés con respecto } \\
\text { a la intervención del } \\
\text { interlocutor interrumpido. } \\
\text { Estrategia discursiva para } \\
\text { expresar afecto, empatía o } \\
\text { solidaridad. } \\
\text { Contribuyen al desarrollo } \\
\text { de la interacción: cubren } \\
\text { huecos de información, } \\
\text { (re)elaboran el tema del } \\
\text { mensaje interrumpido, } \\
\text { introducen juicios de valor, } \\
\text { solicitan un mayor número } \\
\text { de datos sobre los temas. }\end{array}$ & $\begin{array}{l}\text { Actos de habla competitivos. } \\
\text { Se basan en el poder y el } \\
\text { control discursivos. } \\
\text { Intervenciones intrusivas, } \\
\text { inapropiadas o descorteses. } \\
\text { Inserciones incoherentes } \\
\text { con los comentarios del } \\
\text { interlocutor interrumpido. } \\
\text { Estrategia discursiva para } \\
\text { expresar aversión, antipatía y, } \\
\text { ocasionalmente, constituyen } \\
\text { un ataque agresivo. } \\
\text { Suponen, con frecuencia, } \\
\text { un cambio temático o la } \\
\text { recuperación de algún tema } \\
\text { anterior. }\end{array}$ & $\begin{array}{l}\text { Se dirigen a las } \\
\text { necesidades contextuales } \\
\text { inmediatas. } \\
\text { Reclaman una } \\
\text { reparación, repetición o } \\
\text { aclaración de la emisión } \\
\text { precedente. } \\
\text { No están orientadas } \\
\text { al dominio discursivo } \\
\text { ni tampoco están } \\
\text { directamente } \\
\text { relacionadas con } \\
\text { el objeto de la } \\
\text { conversación. } \\
\text { En ocasiones, se asocian } \\
\text { a los ruidos. Una vez } \\
\text { resuelto el “accidente } \\
\text { conversacional”, el } \\
\text { discurso retorna al } \\
\text { estado previo a la } \\
\text { interrupción. }\end{array}$ \\
\hline \multicolumn{3}{|c|}{ GESTIÓN TEMÁTICA } \\
\hline Relevancia & Estatus informativo & Éxito \\
\hline $\begin{array}{l}\text { Relevantes: pertinentes con } \\
\text { el turno anterior del adulto. } \\
\text { Semirrelevantes: relacionadas } \\
\text { con turnos anteriores del } \\
\text { niño, pero no del adulto. } \\
\text { Irrelevantes: sin relación con } \\
\text { ideas previas. }\end{array}$ & $\begin{array}{l}\text { Remáticas: información } \\
\text { nueva. } \\
\text { Temáticas: información } \\
\text { antigua. }\end{array}$ & $\begin{array}{l}\text { Exitosas: atendidas por el } \\
\text { adulto. } \\
\text { No-exitosas: ignoradas } \\
\text { por el adulto. }\end{array}$ \\
\hline
\end{tabular}




\section{METODOLOGÍA}

\subsection{Participantes}

No se puede perder de vista que el SA es una enfermedad catalogada entre las raras, minoritarias o poco prevalentes -afecta aproximadamente a 2,6 por 10.000 personas ( $c f$. Fombonne, 2005) - y que este hecho dificultó la homogeneización de las características que presentan las muestras comparadas. Concretamente, en este estudio participaron cuarenta hablantes nativos de español, veinte de ellos con SA y otros veinte con desarrollo típico (DT). El diagnóstico clínico de SA fue confirmado por los psicólogos que asistieron en la recogida de datos. Con el correspondiente consentimiento informado, las interacciones fueron grabadas para el posterior análisis de los materiales orales.

Con respecto al grupo patológico, catorce sujetos con SA eran naturales de Asturias (España), mientras que los seis restantes procedían de Valencia (España). Gracias a la colaboración de las asociaciones asturiana y valenciana de SA, los datos fueron obtenidos de forma presencial en ambas zonas geográficas. La edad cronológica de los pacientes con SA se situó entre los 6 y los 15 años $(M=11.15)$; diecinueve de ellos eran de sexo masculino y solo incluimos la grabación de una paciente de sexo femenino. En el momento de la recogida de datos, los sujetos con SA no se encontraban bajo tratamiento farmacológico alguno.

Los veinte participantes con DT eran naturales de Almería (España), a excepción de una hablante de origen mexicano. El grupo control quedó formado por once hombres y nueve mujeres con edades cronológicas comprendidas entre los 12 y los 13 años $(M=12.35)$. De acuerdo con los datos proporcionados por el Departamento de Orientación Psicopedagógica del IES Argar (Almería), lugar donde fueron tomadas las grabaciones -también de forma presencial-, ninguno de los sujetos presentaba problemas psicológicos o neurológicos.

\subsection{Instrumento}

De cara a la recogida de los datos orales, se diseñó un Apoyo Gráfico para el Entrenamiento Pragmático (AGEP), con objeto de motivar un discurso oral semiespontáneo e inducido a partir de tareas basadas en la descripción de imágenes. El instrumento quedó integrado por seis fichas ilustradas compuestas por veinticuatro viñetas con contenidos específicos y consignas independientes. Por tanto, la función de las imágenes fue la propia de un guión conversacional. 
Interrupciones y solapamientos en el discurso oral de hablantes con y sin síndrome de Asperger / F. J. RodríGUez MUÑNOZ

\subsection{Procedimiento}

Con anterioridad a las grabaciones, se proporcionó las siguientes instrucciones generales a los participantes:

Te voy a mostrar una serie de fichas con viñetas y te haré unas preguntas muy sencillas sobre lo que observas en cada una de ellas. Por ejemplo, te pediré que te pongas en el lugar de algunos personajes o que me cuentes una pequeña historia a partir de los dibujos. Intenta, siempre que puedas, referirte al número de la viñeta que estás comentando.

A continuación, se mostró cada una de las fichas y se planteó las cuestiones que debían responderse oralmente.

\subsection{Corpus}

El corpus que respalda la presente investigación se divide en dos subcorpus. El primero, el corpus diferencial, está formado por veinte grabaciones en audio procedentes de hablantes con DT y tiene una duración de 97.7 minutos. El segundo, el que pertenece a los sujetos diagnosticados con SA, consta igualmente de veinte grabaciones pero, en este caso, en formato audiovisual y con una duración de 149.3 minutos (Rodríguez Muñoz, 2014). Los datos orales fueron transcritos en ortografía ordinaria a partir de las convenciones básicas del análisis conversacional (ver Anexo).

\subsection{Plan de análisis de datos}

En primer lugar, debido al tamaño muestral, se localizó manualmente en este corpus oral los turnos de habla simultánea de posibles candidatos a constituir solapamientos o interrupciones. Una vez efectuada una primera distinción entre ambos tipos de transiciones, se procedió al recuento de cada tipología, a fin de determinar la existencia de diferencias cuantitativamente significativas entre los solapamientos y las interrupciones que produjeron ambos grupos en su discurso oral.

A continuación, se tuvo en cuenta la taxonomía que se propone en este estudio para el análisis de la interrupción, en la cual se aplicaron con dos principios pragmático-interactivos como la cooperación conversacional y la gestión temática. En ambos casos se asignó el valor correspondiente a cada una de las interrupciones de este corpus. En relación con el primer criterio aplicado, se distinguieron las in- 
terrupciones cooperativas, no cooperativas y neutras; mientras que, en el segundo, se atendió a la relevancia, al estatus informativo y al éxito que tienen los mensajes interruptores en el discurso oral de los informantes.

Además de las diferencias cuantitativas en términos de frecuencia absoluta de los solapamientos y las interrupciones en ambos grupos, la intención fue establecer distinciones de tipo cualitativo a partir de la caracterización de los distintos tipos de interrupción, con miras a analizar el nivel de cooperación que los participantes alcanzan durante el intercambio oral, por un lado, y la naturaleza de las contribuciones que éstos realizan en el proceso de la gestión temática, por otro.

Dado que la diferencia en el tiempo de grabación de cada subcorpus lleva a pensar que los sujetos con SA hablaron más, y ello aumenta las probabilidades de que produzcan más solapamientos e interrupciones que los participantes con DT, se completaron los datos del análisis cuantitativo a partir de dos índices generales; a saber: $\mathrm{SxP}$ (solapamientos por número de palabras) e IxP (interrupciones por número de palabras). En este último caso, aparte del índice general, se distinguió un valor específico en función de los tipos de interrupciones: cooperativas (ICxP), no cooperativas (INCxP), relevantes (IRxP), irrelevantes (IIxP), semirrelevantes (ISRxP), remáticas (IRMxP), temáticas (ITxP), exitosas (IExP) y no-exitosas (INExP). Para el cálculo, se dividió el número de solapamientos, interrupciones y clases de interrupciones entre la cantidad de palabras de que constan las interacciones orales de ambos grupos y, acto seguido, multiplicamos el resultado por mil.

Este análisis establece tres grados de significación en función de las diferencias cuantitativas entre los índices de solapamientos, interrupciones y tipos de interrupciones por número de palabras que se producen entre el grupo de hablantes con DT y SA: i) poseen un alto grado de significación los valores $>1$ atendiendo a la desviación estándar $(D E)$ del promedio $(M)$; ii) les corresponde un bajo grado de significación a los valores $>1$ que solo tienen en cuenta la desviación con respecto al grupo control (DT); y iii) no son significativos los valores $<1$. Dicho de otro modo, se consideró que los índices del grupo SA que se incrementan 1 punto o más de los que le corresponden al grupo DT ya son atípicos y, por tanto, tendrían un grado bajo de significación. No obstante, aquellos índices que exceden en 1 punto o más la desviación estándar de la media de ambos grupos serían altamente significativos.

\section{RESULTADOS}

\subsection{Hablantes con desarrollo típico}

Tras el recuento, se registraron hasta 24 solapamientos en las interacciones orales que se mantuvo con los sujetos sin problemas del desarrollo. Si se tiene en cuenta 
el número total de palabras correspondiente a las interacciones orales de este grupo, 8.883, se calculó un índice de SxP igual a 2,702.

Asimismo, se produjeron 33 interrupciones de distinto tipo, que se reparten como refleja la Figura 1: cooperativas $(33$; $\mathrm{ICxP}=3,715)$, relevantes $(28$; $\mathrm{IRxP}=$ $3,152)$, semirrelevantes $(5 ; \operatorname{ISRxP}=, 563)$, remáticas $(27 ; \mathrm{IRMxP}=3,04)$, temáticas $(6 ; \mathrm{ITxP}=, 675)$, exitosas $(23 ; \mathrm{IExP}=2,589)$ y no-exitosas $(10 ; \mathrm{INExP}=1,126)$. El valor general relativo a IxP fue de 3,715.

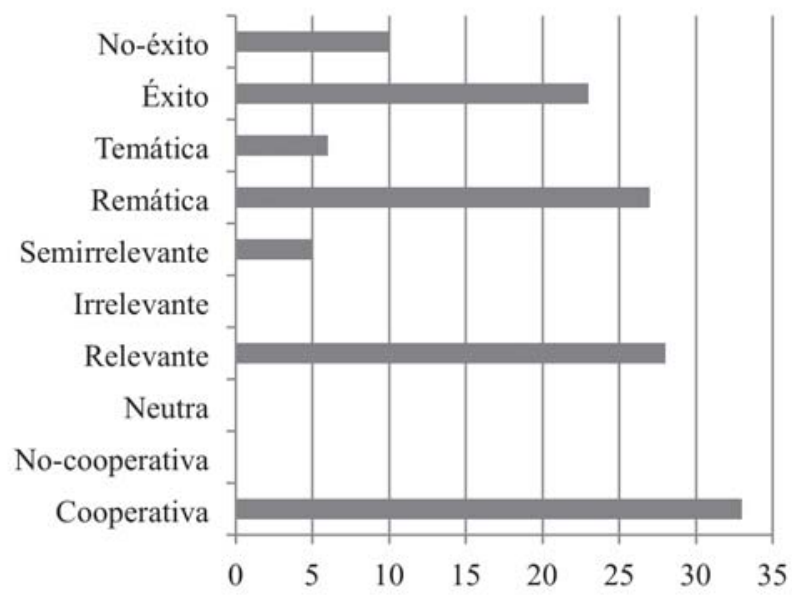

Figura 1. Frecuencia absoluta de interrupciones según su tipología en el discurso oral de hablantes con DT.

Según los datos anteriores, el número de interrupciones supera al de los solapamientos en nuestra muestra de hablantes con DT. Como se dijo, solo se consideró un solapamiento cuando tiene lugar el habla simultánea entre las emisiones de un informante y el entrevistador en los casos en que alguno de éstos pronuncia un sonido fático o alguna expresión lingüística que cumple la misma función comunicativa; es decir, garantizar la eficacia y el control del contacto en la transmisión de la información. Tal es el caso de la expresión uhum (1) cuando la hablante identificada como TCC inicia su intervención solapando el sonido fático emitido por E:

(1) TCC: ese también quiere ir a comprar, pero no- no tiene di- no tiene dinero $₫$

E: [uhum]

TCC: $[$ o(en la seis $)$ o] // va a coger dinero $\$$

E: uhum $₫$ 
La simultaneidad en la emisión del habla que se observa en (1) es un proceso distinto al que tiene lugar cuando se está ante un mensaje propiamente interruptor. En (2) las interrupciones de MMM se incluyen entre las de tipo cooperativo y se interpretó que el estatus informativo es antiguo, por recuperar la información que fue negada en turnos anteriores por la hablante:

(2) E: vale, ¿crees que: podrían ser cuatro niños diferentes viendo lo mismo en la tele? / MMM: no (SONRÍE) $\$$

E: por [ejemplo: $\rightarrow$ o(un) $\left.{ }^{\circ}\right]$

MMM: [bueno, sí $\{$ sí 1 , sî $\$$

E: : ¿sí? \$

MMM: sí $\$$

E: por ejemplo, ¿̇un partido de fútbol? /

MMM: o(pos)o ((¿`cómo)) un partido de fútbol? \$

E: ¿podrían ser cuatros niños diferentes que estuvieran viendo el mismo partido de fútbol? $\$$

MMM: no, (E SONRÍE) no sé $\rightarrow$ (SONRÍE) /

E: que por ejemplo: / el primero reaccione así porque su equipo [va perdiendo] MMM: [jah!, sí, sí,l sí $\$$

E: ¿¿sí? \$

MMM: sí (E Y MMM SE RÍEN) $\$$

Es difícil valorar el grado de pertinencia que tiene la aparición del mensaje interruptor que emite la informante en relación con los turnos inmediatamente precedentes que corresponden al adulto. Las interrupciones de MMM no están directamente asociadas a los enunciados de $\mathrm{E} y$, aunque la informante tenga parcialmente en cuenta esas intervenciones antes de interrumpir y cambiar de opinión, se consideró que ambas son semirrelevantes por guardar una mayor relación semántica con lo que ella misma expresó en turnos previos al cambio de opinión.

El ejemplo (3), perteneciente a la misma hablante, también revela un estado informativo antiguo, ya que el mensaje interruptor se resuelve por medio de una repetición exacta de las palabras pronunciadas por E en el turno anterior:

(3) E: que le cuesta [trabajo andar]

MMM: [sí, que le cuestal trabajo andar $\$$

En (4) se encontró un caso paradigmático de un tipo de mensaje interruptor que, pese a ser cooperativo, no triunfa. Además, la emisión se caracteriza por ser reiterativa o, dicho de otro modo, es temática por incorporar información ya dicha. Se trata de un mensaje semirrelevante que, semánticamente, remite a un 
Interrupciones y solapamientos en el discurso oral de hablantes con y sin síndrome de Asperger / F. J. RodRíGUEz MuÑoz

enunciado precedente del informante DBP:

(4) E: $[(($ vale, $))]$ si tú gastaras esa broma, ¿̇también te reirías? /

DBP: yo pienso que sí $\$$

E: vale, [y si te la gastaran=]

DBP: [o(claro que sí)o]

E: $=$ a ti, ¿̇cómo reaccionarías? $\$$

DBP: $\circ(\mathrm{mal}) \circ \S$

En (5) la hablante NFC interrumpe a E sin dejar que el adulto termine su emisión. A pesar de esta anticipación, justificada por la impulsividad de la niña a lo largo de toda la interacción, la intervención es cooperativa y está orientada al objeto de la conversación. Aun así, se consideró que el mensaje es semirrelevante, y el estado de la información, antiguo, ya que en turnos anteriores, por iniciativa de E, se resuelve que el personaje de las viñetas que asusta a un niño haciéndose pasar por tiburón es una persona disfrazada y no un selacio marino:

(5) E: no era un tiburón (SE RíE) /

NFC: es verdad, que es verdad (SORPRENDIDA) /

E: claro, era un niño que [se había hecho]

NFC: [se pensabal la niña que era $\rightarrow \$$

E: claro, / y entonces le ha gasta(d)o una broma $₫$

NFC: claro, y s'ha enfada(d)o $\$$

Por último, en (6) se encontró el tipo de mensaje interruptor más frecuente en esta muestra de hablantes sin patología, caracterizado por reunir las condiciones de cooperación, relevancia, estado informativo nuevo y éxito:

(6) E: :puede ser que esté esperando a que meta [en el partido el gol?]

VBM: [algún gol, sí,] en el tercero que haya perdido su equipo y en el cuarto pues que se ría de la gente o algo así (RIÉNDOSE) $₫$

\subsection{Hablantes con sindrome de Asperger}

Según los cálculos, en primer lugar, se producen 114 solapamientos en el discurso oral de los participantes afectados por SA. Si se divide esta cifra entre 10.397, se obtiene un índice de SxP igual a 10,965.

En segundo lugar, se contó hasta 62 interrupciones, que se reparten como refleja la Figura 2: cooperativas $(51 ; \mathrm{ICxP}=4,905)$, no-cooperativas $(11 ; \mathrm{INCxP}=$ $1,058)$, relevantes $(44 ; \operatorname{IRxP}=4,232)$, irrelevantes $(14$; IIxP=1,347), semirrelevantes $(4 ; \mathrm{ISRxP}=0,385)$, remáticas $(54 ; \mathrm{IRMxP}=5,194)$, temáticas $(8 ; \mathrm{ITxP}=$ 
0,769), exitosas (51; IExP $=4,905)$ y no-exitosas $(11 ; \mathrm{INExP}=1,058)$. En este caso, el índice IxP equivale a 5,963.

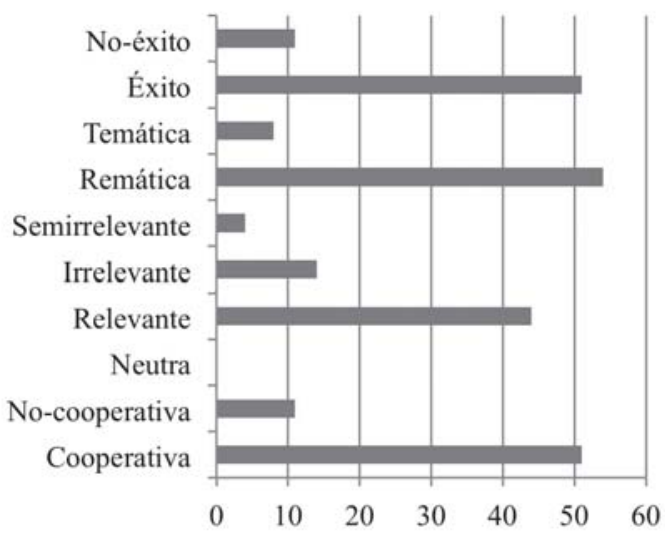

Figura 2. Frecuencia absoluta de interrupciones según su tipología en el discurso oral de hablantes con SA.

De acuerdo con los datos recabados y al igual que se apuntó para la muestra de hablantes sin problemas del desarrollo, el número de solapamientos es superior al de las interrupciones en el grupo de sujetos diagnosticados con SA. En (7) el participante identificado con las iniciales APC emite la expresión coloquial guay, a la vez que el entrevistador le explica las instrucciones a las que habrá de atenerse durante el intercambio:

(7) E: venga, mira, te enseño estas viñetas, [ïvale?=]

APC: [gua::y^]

E: =y tú tienes que decirme / cómo crees que se siente el niño en cada una de las viñetas, me dices en la viñeta uno, / ¿enten[diste?]

En relación con los procesos de interrupción que tienen lugar durante los intercambios mantenidos con los individuos afectados por SA -así como también sucedía en la muestra de hablantes con DT-, las cuatro condiciones atribuidas a la interrupción que concurren con mayor frecuencia en el discurso oral son: cooperativa, relevante, remática y exitosa.

Los ejemplos (8) y (9), de contenido muy similar, sirven para ilustrar el proceso de interrupción más habitual en hablantes con y sin patología. Tanto DGM como FMS inician su respuesta antes de que $\mathrm{E}$ termine de formular la pregunta. El mensaje interruptor de ambos participantes es cooperativo y la información que 
Interrupciones y solapamientos en el discurso oral de hablantes con y sin síndrome de Asperger / F. J. RodRíGUEz MuÑoz

proporcionan es nueva y relevante. Además, se debe mencionar que las interrupciones resultan exitosas, ya que son atendidas por el interlocutor:

(8) E: ¿cómo te sientes cuando [te gastan bromas?]

DGM: [enfadado]

E: enfada(d)o, ¿̨te gusta gastar bromas tú? /

DGM: psh, $\left(\left({ }^{\circ}(\text { bueno })^{\circ}\right)\right)$ (TONO DUBITATIVO) $\$$

(9) E: vale, ¿̨cómo te sentirías tú si te [gastaran esa?]

FMS: [enfadado]

E: muy bien, / ipues ya está! $\$$

Según estas intuiciones preliminares, existirían diferencias cualitativas entre los tipos de mensajes interruptores presentes en las dos muestras de hablantes comparados. Por ejemplo, frente a la ausencia total de interrupciones no-cooperativas en el discurso oral que protagonizan los hablantes con DT, en (10) se encontró varias interrupciones que se caracterizaron de esta forma:

(10) E: y y, por ejemplo, mira, la expresión [de la caral

APC: [guau, guaul (HACE LA FORMA DE UN PERRO CON LA MANO IZQUIERDA Y EMITE LADRIDOS MIENTRAS MIRA A LA CÁMARA)

E: [Álvaro, Álvaro]

APC: [guau, guau]

E: [Álvaro, Álvaro,] mira, ¿̨la expresión de la cara de esta mm viejecita, qué diría? $\$$

En el ejemplo (10), los turnos de APC constituyen interrupciones con carácter intrusivo y son actos no colaborativos, ya que entorpecen el desarrollo natural de la interacción. APC forma con sus manos la figura de un perro y, sin ninguna razón que lo conduzca a ello, empieza a emitir ladridos. Ante dicha situación, el entrevistador llama la atención al informante en repetidas ocasiones.

Otra de las diferencias cualitativas que se encontró en relación con los tipos de interrupción es que los mensajes interruptores son menos relevantes en el discurso oral de la muestra de hablantes con SA. En efecto, frente a la total ausencia de interrupciones irrelevantes en las interacciones de los participantes sin problemas del desarrollo, ahora se encontró quince interrupciones de este tipo, por no guardar relación alguna con los enunciados precedentes. En (11) APC, de nuevo, comienza a emitir ruidos injustificadamente, pues obviamente no tienen carácter fático o de control de contacto. En este sentido, la interrupción resulta completamente irrelevante para el propósito comunicativo, toda vez que entorpece el desarrollo fluido del intercambio: 
RLA. Revista de Lingüística Teórica y Aplicada, 53 (1), I Sem. 2015

(11) E: [muy bien, mira, Álvaro]

APC: [ah::, ah::, uh::, ah::] (EMITE RUIDOS Y HACE MOVIMIENTOS CON LA BOCA)

E: [Álvaro, Álvaro, Álvaro,] mira otra, que esta es muy chuli, [ya verás, ven$\mathrm{ga}=1$

APC: \hala $\uparrow$, nadando $\uparrow$, genial $\uparrow\rfloor$

$\mathbf{E}:=$ mira, ¿qué pasa en estas viñetas?, / en la-, mira, en la veinte y en la veintiuno, ¿en la veinte qué pasa? I

En el extracto (12), el hablante DCF sencillamente ignora la emisión de E. En consecuencia, se interpretó que el mensaje interruptor que introduce es de carácter semirrelevante por estar semánticamente asociado a otros turnos anteriores:

(12) E: ¿en cada una?, dime, [en la uno]

DCF: [bueno,] en la uno, mal $\S$

E: [mal, ((:por qué?))]

DCF: [en la dos,] nervioso, en la tres, triste, y en la cuatro se siente: $\mathrm{mm}$ contento (MOVIENDO LA CABEZA) $₫$

\subsection{Hablantes con desarrollo típico y síndrome de Asperger}

Se consideró que el valor estándar de solapamientos e interrupciones por número de palabras es el que les corresponde a los participantes del grupo control; en este sentido, se estimó que son significativos los valores $\mathrm{SxP}$, IxP y sus derivados que el grupo de hablantes con SA supera al menos en 1 punto. De acuerdo con esta pauta, a excepción de los índices relativos a las interrupciones semirrelevantes (IS$\mathrm{RxP})$, temáticas (ITxP) y no-exitosas (INExP) por número de palabras, todos los demás tendrían un grado bajo de significación; a saber, los índices que oponen las características cooperación/no cooperación (ICxP/INCxP), así como relevancia/ irrelevancia (IRxP/IIxP).

No obstante, la Tabla II recoge los estadísticos descriptivos relativos a la media $(M)$ y a la desviación estándar $(D E)$. De este modo, si se tiene en cuenta la $D E$, los índices que exceden en más de 1 punto a los del grupo control son SxP, IxP, IRMxP e IExP, y poseerían un grado alto de significación. 
Interrupciones y solapamientos en el discurso oral de hablantes con y sin síndrome de Asperger / F. J. RodríGUEZ MUÑNOZ

Tabla II. Solapamientos, interrupciones y tipos de interrupciones más o menos significativos en el discurso oral de hablantes con y sin síndrome de Asperger.

\begin{tabular}{lrrrrr}
\hline Tipos de transiciones & DT & SA & $\boldsymbol{M}$ & DE & $\begin{array}{r}\text { Grado } \\
\text { de sig. }\end{array}$ \\
\hline SxP solapamientos & 2,702 & 10,965 & 6,83350 & 4,525831 & $* *$ \\
\hline IxP interrupciones & 3,715 & 5,963 & 4,83900 & 1,231280 & $* *$ \\
\hline ICxP interrupciones cooperativas & 3,715 & 4,905 & 4,31000 &, 651790 & $*$ \\
\hline INCxP interrupciones no cooperativas & 0 & 1,058 &, 52900 &, 579490 & $*$ \\
\hline IRxP interrupciones relevantes & 3,152 & 4,232 & 3,69200 &, 591540 & $*$ \\
\hline IIxP interrupciones irrelevantes & 0 & 1,347 &, 67350 &, 737782 & $*$ \\
\hline ISRxP interrupciones semirrelevantes &, 563 &, 385 &, 47400 &, 097495 & - \\
\hline IRMxP interrupciones remáticas & 3,04 & 5,194 & 4,11700 & 1,179794 & $* *$ \\
\hline ITxP interrupciones temáticas &, 675 &, 769 &, 72200 &, 051486 & - \\
\hline IExP interrupciones exitosas & 2,589 & 4,905 & 3,74700 & 1,268525 & $* *$ \\
\hline INExP interrupciones no-exitosas & 1,126 & 1,058 & 1,09200 &, 037245 & - \\
\hline
\end{tabular}

* Grado alto, ${ }^{* *}$ Grado bajo, Grado nulo.

Más gráficamente, la Figura 3 muestra las diferencias cuantitativas que se producen a propósito de los índices que se calcularon para los solapamientos, las interrupciones y las clases de interrupciones por número de palabras en las interacciones orales que protagonizan los dos grupos de sujetos comparados.

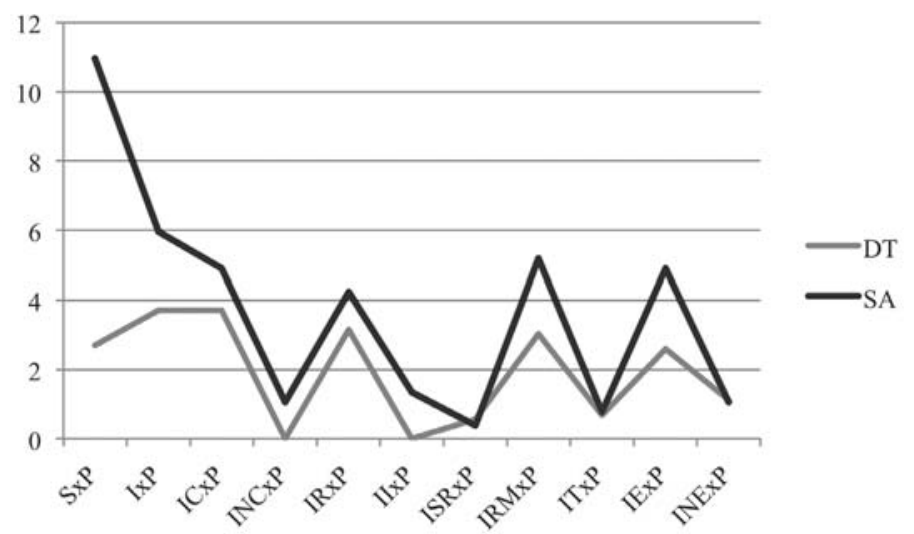

Figura 3. Diferencias cuantitativas de los índices relativos a los solapamientos, las interrupciones y los tipos de interrupciones por número de palabras en el discurso oral de hablantes con DT y SA. 


\section{DISCUSIÓN}

Tras el análisis de las interrupciones y los solapamientos en las interacciones de hablantes con SA y sin problemas del desarrollo, se comprobó que existe un predominio cuantitativamente significativo en el discurso oral de los primeros al efectuar este tipo de transiciones. Por una parte, el número de solapamientos por palabra que producen es muy superior $(\mathrm{S} x \mathrm{P}=10,965)$ en comparación con el que les corresponde a los participantes con DT $(S \mathrm{xP}=2,702)$. Lo mismo se puede señalar a propósito del número de interrupciones por palabra que producen los hablantes afectados por $\mathrm{SA}(\mathrm{IxP}=5,963)$, frente a la muestra sin patología $(\mathrm{IxP}=3,715)$.

Por otra parte, se constató que los mensajes interruptores que emiten los sujetos con SA son cualitativamente menos cooperativos si los comparamos con los de los participantes con DT. Además, en ninguno de los dos grupos analizados se producen interrupciones con carácter neutro; es decir, condicionadas por accidentes conversacionales que, a su vez, suelen estar relacionados con el contexto y dirigidos a resolver las necesidades inmediatas de la comunicación.

De acuerdo con el grado de relevancia, cabe destacar que existe una representación de interrupciones irrelevantes en los intercambios orales protagonizados por los hablantes afectados por SA, mientras que esta clase se encuentra ausente en el grupo control. Sin embargo, las interrupciones de naturaleza semirrelevante están prácticamente equiparadas en el discurso oral de ambos grupos.

Las interrupciones que se denominaron remáticas -por aportar información nueva- y las exitosas -las que son respondidas por el interlocutor- poseen un alto grado de significación -esto es, son mucho más numerosas- en el grupo de participantes con SA. Es preciso advertir que esta segunda circunstancia no depende totalmente del emisor, pues el receptor (adulto) es quien finalmente decide atender o no la interrupción de su interlocutor. Se interpreta que, en tales situaciones, existe una mayor predisposición por parte del entrevistador a considerar los mensajes interruptores que emiten los informantes con patología. Es decir, se produce una exaltación de la máxima de cortesía, relacionada con un exceso de deferencia ( $c f$. Moreno Campos, 2006), de la que es responsable último el entrevistador. Goffman (1967) ya señalaba dos clases de reglas encaminadas a preservar la imagen: la regla del respeto hacia uno mismo y la regla de la atención. La segunda es la que el entrevistador aplica sistemáticamente en las interacciones orales protagonizadas por los hablantes con SA.

\section{CONCLUSIÓN}

Tal como se preveía, los solapamientos y las interrupciones -concebidos como tipos de transición entre turnos conversacionales- han demostrado ser categorías 
significativas para el estudio del estilo conversacional de los hablantes diagnosticados con SA. El análisis presentado se hace atractivo por los indicadores referentes a la conducta oral que ofrece, sobre todo, de cara a la intervención comunicativa de las personas con SA. En este sentido, no se puede pasar por alto que la patología objeto de estudio constituye, en términos lingüísticos, un trastorno pragmático de la comunicación; de manera que es preciso reivindicar la reorientación de aquellos métodos de intervención que se ocupan de los componentes formales del lenguaje, para darle prioridad a los aspectos pragmáticos. Más específicamente, es en la dimensión interactiva de la pragmática donde se concentra el mayor número de déficits lingüísticos característicos del SA, área en la que se sitúa el estudio de los solapamientos y las interrupciones ( $c f$. Rodríguez Muñoz, 2013).

Por último, estos resultados sugieren que será preciso propiciar en estos hablantes aportaciones más relevantes y cooperativas durante los intercambios orales, evitando así contribuciones más irrelevantes o menos colaborativas. Además, los datos proporcionados en esta investigación necesitan ser completados por otros análisis encaminados a precisar aún más los patrones conversacionales que definen el SA; solo de ese modo será posible avanzar en el conocimiento de dicha patología.

\section{REFERENCIAS}

Bañón, Antonio M. (1997). La interrupción conversacional. Propuestas para su análisis pragmalingüistico. Málaga: Universidad de Málaga.

Beattie, Geoffrey W. (1981). Interruption in conversational interaction and its relation to the sex and status of the interactants. Linguistics, 19, 15-35.

Beattie, Geoffrey W. (1982). Turn-taking and interruption in political interviews: Margaret Thatcher and Jim Callaghan compared and contrasted. Semiotica, 39 (1/2), 93-114.

Bennett, Adrian. (1981). Interruption and the interpretation of conversation. Discourse Processes, 4 (2), 171-188.

Bogetic, Ksenija. (2011). Interruptions and the dyadic co-narration of shared experiences in English and Serbian conversation. Language \& Communication, 31 (4), 318-328.

Burgos, Félix Manuel. (2007). Co-construcciones en español: ¿Cooperación por medio de una interrupción?, Forma y Función, 20, 13-39.

Dindia, Kathryn. (1987). The effects of sex of subject and sex of partner on interruptions. Human Communication Research, 13 (3), 345-371.

Dunn, Judy y Marilyn Shatz. (1989). Becoming a conversationalist despite (or because of) having a sibling. Child Development, 60 (2), 399-410.

Edelsky, Carole. (1981). Who's got the floor? Language in Society, 10 (5), 383-421. 
Ervin-Tripp, Susan M. (1979). Children's verbal turn-taking. En Elinor Ochs y Bambi B. Schieffelin (eds.), Developmental pragmatics (pp. 391-414). Londres: Academic Press.

Farina, Amerigo. (1960). Patterns of role dominance and conflict in parents of schizophrenic patients. Journal of Abnormal and Social Psychology, 61 (1), 3138.

Farley, Sally D., Amie M. Ashcraft, Mark F. Stasson y Rebecca L. Nusbaum. (2010). Nonverbal reactions to conversational interruption: A test of complementarity theory and the status/gender parallel. Journal of Nonverbal Behavior, 34 (4), 193-206.

Ferguson, Nicola. (1977). Simultaneous speech, interruptions and dominance. British Journal of Social and Clinical Psychology, 16 (4), 295-302.

Fombonne, Eric. (2005). Epidemiology of autistic disorder and other pervasive developmental disorders. Journal of Clinical Psychiatry, 66 (Supl. 10), 3-8.

French, Peter y John Local. (1983). Turn-competitive incomings. Journal of Pragmatics, 7 (1), 17-38.

Gallardo, Beatriz. (1993). La transición entre turnos conversacionales: silencios, interrupciones y solapamientos. Contextos, XI (21-22), 189-220.

Gallardo, Beatriz. (2003). El diálogo entre personas. En Joaquim Llisterri y María Jesús Machuca (eds.), Sistemas de diálogo (pp. 5-90). Bellaterra/Soria: Universidad Autónoma de Barcelona/Fundación Duques de Soria.

Gallardo, Beatriz. (2006). Más allá de las palabras y su estructura: las categorías del componente pragmático. En Elena Garayzábal (ed.), Lingüistica clínica y logopedia (pp. 81-196). Madrid: Antonio Machado Libros.

Gallois, Cynthia y Norman N. Markel. (1975). Turn taking: social personality and conversational style. Journal of Personality and Social Psychology, 31 (6), 1134-1140.

Goffman, Erving. (1967). Interaction ritual. Chicago, IL: Aldine Publishing Co.

Goldberg, Julia A. (1990). Interrupting the discourse of interruptions. An analysis in terms of relationally neutral, power- and rapport-oriented acts. Journal of Pragmatics, 14 (6), 883-903.

Grice, H. Paul. (1975). Logic and conversation. En Peter Cole y Jerry L. Morgan (eds.), Syntax and Semantics, vol. 3: Speech Acts (pp. 41-58). Nueva York: Academic Press.

Hetherington, E. Mavis, Roger J. Stouwie y Eugene H. Ridberg. (1971). Patterns of family interaction and child-rearing attitudes related to three dimensions of juvenile delinquency. Journal of Abnormal Psychology, 78 (2), 160-176.

Houtkoop, Hanneke y Harrie Mazeland. (1985). Turns and discourse units in everyday conversation. Journal of Pragmatics, 9 (5), 595-619.

Jacob, Theodore. (1974). Patterns of family conflict and dominance as a function of child age and social class. Developmental Psychology, 10 (1), 1-12. 
Jacob, Theodore. (1975). Family interaction in disturbed and normal families: A methodological and substantive review. Psychological Bulletin, 82 (1), 33-65.

Kennedy, Carol W. y Carl T. Camden. (1983). A new look at interruptions. Western Journal of Speech Communication, 47 (1), 45-58.

Kollock, Peter, Pepper Blumstein y Philip Schwartz. (1985). Sex and power in interaction: Conversational privileges and duties. American Sociological Review, 50, 34-46.

Liddicoat, Anthony J. (2007). An introduction to conversation analysis. Londres: Continuum.

Meltzer, Leo, William N. Morris y Donald P. Hayes. (1971). Interruption outcomes and vocal amplitude: explorations in social psychophysics. Journal of Personality and Social Psychology, 18 (3), 392-402.

Menz, Florian y Ali Al-Roubaie. (2008). Interruptions, status and gender in medical interviews: the harder you brake, the longer it takes. Discourse \& Society, 19 (5), 645-666.

Mishler, Elliot G. y Nancy E. Waxler. (1968). Interaction in families: An experimental study of family processes and schizophrenia. Nueva York: John Wiley.

Moreno Campos, Verónica. (2006). Afasia y tempo dialógico: el índice de participación conversacional. En Beatriz Gallardo, Carlos Hernández y Verónica Moreno (eds.), Lingüistica clínica y neuropsicología cognitiva. Actas del Primer Congreso Nacional de Lingüistica Clinica. Vol. 1: Investigación e intervención en patologías del lenguaje (pp. 87-106). Valencia: Universidad de Valencia.

Murata, Kumiko. (1994). Intrusive or co-operative? A cross-cultural study of interruption. Journal of Pragmatics, 21 (4), 385-400.

Murray, Stephen O. (1987). Power and solidarity in 'interruption': A critique of the Santa Barbara School conception and its application by Orcutt and Harvey (1985). Symbolic Interaction, 10 (1), 101-110.

O'Reilly, Michelle. (2008). What value is there in children's talk? Investigating family therapists' interruptions of parents and children during the therapeutic process. Journal of Pragmatics, 40 (3), 507-524.

Orcutt, James D. y Lynn Kenneth Harvey. (1985). Deviance, rule-breaking and male dominance in conversation. Symbolic Interaction, 8 (1), 15-32.

Riskin, Jules M. D. y Elaine E. Faunce. (1972). An evaluative review of family interaction research. Family Process, 11 (4), 365-456.

Rodríguez Muñoz, Francisco J. (2012). La conciencia pragmática de adultos con síndrome de Asperger. Revista de Logopedia, Foniatría y Audiología, 32 (1), 21-31.

Rodríguez Muñoz, Francisco J. (2013). Evaluación pragmática de niños con sindrome de Asperger. Münich: Lincom.

Rodríguez Muñoz, Francisco J. (2014). Corpus oral de hablantes con desarrollo típico y sindrome de Asperger. Berlín: Logos Verlag. 
Tannen, Deborah. (1981). Nueva York Jewish conversational style. International Journal of the Sociology of Language, 32, 133-149.

West, Candace. (1979). Against our will: Male interruptions of females in crosssex conversations. Annals of the Nueva York Academy of Sciences, 327, 81-97.

\section{ANEXO. Convenciones de transcripción}

$\begin{array}{ll}\text { E: } & \text { Turno perteneciente al entrevistador } \\ / / & \text { Pausa larga simple (1-2 segundos) } \\ \uparrow & \text { Pausa larga doble (2-3 segundos) } \\ - & \text { Entonación ascendente } \\ : & \text { Corte abrupto o autointerrupción } \\ ((\text { Dobles paréntesis })) & \text { Tlargamiento } \\ o()^{\circ} & \text { Transcripción dudosa } \\ {[]} & \text { Volumen bajo } \\ & \text { [ Comienzo de un segmento de habla simultánea } \\ \mathbb{S} & \text { ] Final de un segmento de habla simultánea } \\ = & \text { Encadenamiento de turnos } \\ \text { (VERSALES }) & \text { Final y comienzo de un mismo turno en el que se intercala } \\ \text { Cursiva } & \text { Comentarios del transcriptor }\end{array}$

